\title{
Stability and Bifurcation Analysis of a Singular Delayed Predator-Prey Bioeconomic Model with Stochastic Fluctuations
}

\author{
Yue Zhang and Qingling Zhang \\ Institute of Systems Science, Northeastern University, Shenyang 110819, China \\ Correspondence should be addressed to Qingling Zhang; qlzhang@mail.neu.edu.cn
}

Received 25 August 2014; Accepted 25 November 2014; Published 7 December 2014

Academic Editor: Hongyong Zhao

Copyright ( 2014 Y. Zhang and Q. Zhang. This is an open access article distributed under the Creative Commons Attribution License, which permits unrestricted use, distribution, and reproduction in any medium, provided the original work is properly cited.

This study investigates a singular delayed predator-prey bioeconomic model with stochastic fluctuations, which is described by differential-algebraic equations because of economic factors. The interior equilibrium of the singular delayed predator-prey bioeconomic model switches from being stable to unstable and then back to being stable, with the increase in time delay. The critical values for stability switches and Hopf bifurcations can be analytically determined. Subsequently, the effect of a fluctuating environment on the singular stochastic delayed predator-prey bioeconomic model obtained by introducing Gaussian white noise terms to the aforementioned deterministic model system is discussed. The fluctuation intensity of the population and harvest effort are calculated by Fourier transform method. Numerical simulation results are presented to verify the effectiveness of the conclusions.

\section{Introduction}

In nature, populations do not reproduce instantaneously; rather, it is mediated by certain time delay required for gestation, maturation time, capturing time, or other reasons. Thus, time delays of one type or another have been incorporated into mathematical models of population dynamics. The dynamic relationship between predators and their prey has long been and will continue to be one of the dominant themes in both ecology and mathematical ecology because of its universal existence and importance. The following delayed Leslie-Gower predator-prey system is a type of predator-prey model with time delay:

$$
\begin{gathered}
\dot{x}(t)=r_{1} x(t)\left(1-\frac{1}{K} \int_{-\infty}^{t} F(t-s) x(s) d s\right)-\beta x(t) y(t), \\
\dot{y}(t)=r_{2} y(t)\left(1-\frac{y(t)}{\gamma x(t)}\right),
\end{gathered}
$$

where $r_{1}, r_{2}, K, \gamma$, and $\beta$ are positive constants. When $F(t-$ $s)=\delta(t-s-\tau), \delta$ is the delta function, $\tau$ is a positive constant, and system (1) transforms in the following form with a discrete delay:

$$
\begin{gathered}
\dot{x}(t)=r_{1} x(t)\left(1-\frac{x(t-\tau)}{K}\right)-\beta x(t) y(t), \\
\dot{y}(t)=r_{2} y(t)\left(1-\frac{y(t)}{\gamma x(t)}\right) .
\end{gathered}
$$

The variables $x(t)$ and $y(t)$ denote the population of the prey and the predator, respectively. The parameters $r_{1}$ and $r_{2}$ are the intrinsic growth rates of the prey and the predator, respectively. The value $K$ is the carrying capacity of the prey and $\gamma x(t)$ is a prey-dependent carrying capacity for the predator. The parameter $\gamma$ is a measure of the quality of the prey as food for the predator and $\beta x(t)$ is the predator functional response to prey, which satisfies the usual properties that make system (1) a predator-prey one.

To date, many authors [1-7] have studied the dynamics of predator-prey models with time delay and obtained complex dynamic behavior, such as stability of equilibrium, Hopf bifurcation, and limit cycle. A number of references [8-12] have discussed the dynamic behavior of delayed predatorprey models with harvesting and determined that positive 
equilibrium switches from being stable to unstable and then back to being stable, as the delay increases. In addition, some results about the problem of bifurcation and control on some singular prey-predator bioeconomic models with time delay have been discussed [13-17].

For system (2), Yuan and Song [4] have investigated the existence, direction, and stability of Hopf bifurcation. However, they did not discuss the dynamical behavior of system (2) in terms of harvesting and fluctuation.

In the current paper, harvesting is introduced by the following harvest production function:

$$
Y(t)=E(t) x(t)
$$

This particular form of the Cobb-Douglas production function is the standard harvest production function employed in classical fishery models. This harvest production function is valid in the previous case (trawl) fisheries, where $E(t)$ is the harvest effort (often associated with the number of boats, flow of labor, and capital services devoted to harvesting fish). In addition, this function satisfies the following economic restriction: Net Economic Revenue $($ NER) = Total Revenue (TR) - Total Cost (TC). Based on Model system (2) and the aforementioned economic theory, the following singular bioeconomic model, which consists of two differential equations and an algebraic equation (differential-algebraic equations), was developed:

$$
\begin{gathered}
\dot{x}(t)=r_{1} x(t)\left(1-\frac{x(t-\tau)}{K}\right)-\beta x(t) y(t)-E(t) x(t), \\
\dot{y}(t)=r_{2} y(t)\left(1-\frac{y(t)}{\gamma x(t)}\right), \\
0=E(t)(p x(t)-c)-m,
\end{gathered}
$$

where $x(t), y(t), r_{1}, r_{2}, K, \gamma$, and $\beta$ are the same as those described in model system (2); $p$ is the price of a unit of harvested biomass; $p E(t) x(t)$ is the total revenue; $c$ is the cost of a unit of effort; $c E(t)$ is the total cost; and $m$ is the net economic revenue. The constants are all positive.

In reality, environmental fluctuation is one of the important components of ecological systems within a natural environment. A large part of natural phenomena do not follow the deterministic law; rather, they oscillate randomly around an average value. The deterministic approach has some limitations in mathematical modeling. Thus, accurately predicting the future dynamics of the system is difficult. The stochastic differential equation models play a significant role in various dynamical analyses of systems because they can provide an additional degree of realism compared with their deterministic counterpart [18]. Recently, the stochastic modeling of ecological population systems has gained a great deal of attention from several scientists [19-26]. The following stochastic version model that corresponds to the delayed model system (4) within the fluctuating environment is provided to verify the effect of environmental fluctuation on a delayed singular prey-predator bioeconomic model:

$$
\begin{gathered}
\dot{x}(t)=r_{1} x(t)\left(1-\frac{x(t-\tau)}{K}\right)-\beta x(t) y(t) \\
-E(t) x(t)+\xi_{1}(t), \\
\dot{y}(t)=r_{2} y(t)\left(1-\frac{y(t)}{\gamma x(t)}\right)+\xi_{2}(t), \\
0=E(t)(p x(t)-c)-m+\xi_{3}(t),
\end{gathered}
$$

where the perturbed terms $\xi_{i}, i=1,2,3$, are mutually independent Gaussian white noises characterized by $\left\langle\xi_{i}\right\rangle=0$ and $\left\langle\xi_{i}(t) \xi_{j}\left(t_{1}\right)\right\rangle=\delta_{i j} \delta\left(t-t_{1}\right), i, j=1,2,3$. $\langle\cdot\rangle$ represents the ensemble average due to the effect of fluctuating environment, $\delta_{i j}$ is the Kronecker delta expressing the spectral density of the white noise, and $\delta$ is the Dirac delta function with $t$ and $t_{1}$ being the distinct times.

The rest of the paper is organized as follows. The effect of time delay on the stability of the interior equilibrium of system (4) is investigated in Section 2. The effect of fluctuating environment on the singular stochastic delayed LeslieGower predator-prey bioeconomic model (5) is discussed in Section 3. Numerical simulation results are presented to verify the effectiveness of the conclusions in Section 4. Finally, concluding remarks are given.

\section{Stability of Equilibria and Existence of Hopf Bifurcations}

In this section, we concentrate on the stability switch and Hopf bifurcation phenomenon around the interior equilibrium of model system (4) because of the variation of time delay $\tau$, given that the biological interpretation of the interior equilibrium implies the existence of population and harvest effort.

$P^{*}\left(x^{*}, y^{*}, E^{*}\right)$ denotes the interior equilibrium of model system (4) under positive economic profit of harvesting $m>$ 0 , where $y^{*}=r x^{*}, E^{*}=m /\left(p x^{*}-c\right)$, and $x^{*}$ satisfies the following equation:

$$
p\left(\frac{r_{1}}{k}+\beta \gamma\right) x^{* 2}-\left[c\left(\frac{r_{1}}{k}+\beta \gamma\right)+p r_{1}\right] x^{*}+\left(c r_{1}+m\right)=0 .
$$

If $p r_{1}-c\left(r_{1} / k+\beta \gamma\right)>0$, then two interior equilibria exist when $0<m<m^{*}$, one interior equilibrium exists when $m=m^{*}$, and no interior equilibrium exists when $m=m^{*}$, where $m^{*}=\left[c\left(r_{1} / k+\beta \gamma\right)-p r_{1}\right]^{2} / 4 p\left(r_{1} / k+\beta \gamma\right)$. Based on this discussion, two interior equilibria (denoted by $P_{1}^{*}$ and $P_{2}^{*}$ ) exist when $0<m<m^{*}$. This study only investigated the dynamical behavior of model system (4) at the interior equilibrium $P_{1}^{*}$. Symmetric results on the interior equilibrium $P_{2}^{*}$ can be also obtained.

According to the Jacobian evaluated at the interior equilibrium $P_{1}^{*}$ and the leading matrix in model system (4), the 
characteristic equation of model system (4) at equilibrium $P_{1}^{*}$ can be expressed as follows:

$$
\begin{aligned}
D(\lambda, \tau) & =\operatorname{det}\left(\begin{array}{ccc}
\lambda+\frac{r_{1}}{k} x^{*} e^{-\lambda \tau} & \beta x^{*} & x^{*} \\
-\frac{r_{2} y^{* 2}}{\gamma x^{* 2}} & \lambda+\frac{r_{2} y^{*}}{\gamma x^{*}} & 0 \\
-p E & 0 & -\left(p x^{*}-c\right)
\end{array}\right) \\
& =P(\lambda)+Q(\lambda) e^{-\lambda \tau}=0,
\end{aligned}
$$

where

$$
\begin{gathered}
P(\lambda)=p_{0} \lambda^{2}+p_{1} \lambda+p_{2}, \\
Q(\lambda)=q_{1} \lambda+q_{2}, \\
p_{0}=c-p x^{*}, \\
p_{1}=-\frac{p r_{2}}{\gamma} y^{*}+p x^{*} E^{*}+\frac{c r_{2} y^{*}}{\gamma x^{*}}, \\
p_{2}=\frac{p r_{2}}{\gamma} y^{*} E^{*}-\frac{p r_{2} \beta}{\gamma} y^{* 2}+\frac{c r_{2} \beta y^{* 2}}{\gamma x^{*}}, \\
q_{1}=\frac{c r_{1}}{k} x^{*}-\frac{p r_{1}}{k} x^{* 2}, \\
q_{2}=\frac{c r_{1} r_{2}}{k \gamma} y^{*}-\frac{p r_{1} r_{2}}{k \gamma} x^{*} y^{*} .
\end{gathered}
$$

Theorem 1. If conditions $p_{1}+q_{1}<0, p_{2}+q_{2}<0, q_{1}^{2}+2 p_{0} p_{2}-$ $p_{1}^{2}<0$, and $p_{2}^{2}-q_{2}^{2}>0$ are satisfied, then the equilibrium $P_{1}^{*}$ is asymptotically stable for all $\tau \geq 0$.

Proof. For $\tau=0$, the characteristic equation becomes

$$
p_{0} \lambda^{2}+\left(p_{1}+q_{1}\right) \lambda+p_{2}+q_{2}=0
$$

which has the following roots:

$$
\lambda=\frac{-\left(p_{1}+q_{1}\right) \pm \sqrt{\left(p_{1}+q_{1}\right)^{2}-4 p_{0}\left(p_{2}+q_{2}\right)}}{2 p_{0}} .
$$

Thus, $p_{0}<0$ because $x^{*}>0$ and $E^{*}>0$. Looking at (10), the equilibrium $P_{1}^{*}$ is locally asymptotically stable when both roots are negative real parts, if and only if

$$
p_{1}+q_{1}<0, \quad p_{2}+q_{2}<0 .
$$

For the case of $\tau \neq 0$, equilibrium $P_{1}^{*}$ will be locally asymptotically stable if the roots of $D(\lambda, 0)=0$ are negative real parts and $D(i \omega, \tau) \neq 0$ for every real $\omega$ and $\tau \geq 0$. We assume that, for some values of $\tau>0$, a real number $\omega>0$ exists such that $\lambda=i \omega$ is a root of the characteristic (7). Separating the real and imaginary parts of $D(i \omega, \tau)=0$, the following equation can be obtained:

$$
\begin{gathered}
-p_{0} \omega^{2}+p_{2}+q_{1} \omega \sin (\omega \tau)+q_{2} \cos (\omega \tau)=0, \\
p_{1} \omega+q_{1} \omega \cos (\omega \tau)-q_{2} \sin (\omega \tau)=0 .
\end{gathered}
$$

Simplifying system (12), the following equation could be obtained:

$$
p_{0}^{2} \omega^{4}+\left(p_{1}^{2}-2 p_{0} p_{2}-q_{1}^{2}\right) \omega^{2}+p_{2}^{2}-q_{2}^{2}=0
$$

Based on (13), the following equation can be obtained:

$$
\begin{aligned}
& \omega_{ \pm}^{2} \\
& =\frac{\left(q_{1}^{2}+2 p_{0} p_{2}-p_{1}^{2}\right) \pm \sqrt{\left(q_{1}^{2}+2 p_{0} p_{2}-p_{1}^{2}\right)^{2}-4 p_{0}^{2}\left(p_{2}^{2}-q_{2}^{2}\right)}}{2 p_{0}^{2}} .
\end{aligned}
$$

If

$$
q_{1}^{2}+2 p_{0} p_{2}-p_{1}^{2}<0, \quad p_{2}^{2}-q_{2}^{2}>0
$$

are satisfied, (14) does not have positive solutions. That is, the characteristic (7) does not have pure imaginary roots. Using Rouche's theorem [27], all roots of (7) have negative real parts for all $\tau \geq 0$; that is, the equilibrium $P_{1}^{*}$ is asymptotically stable for all $\tau \geq 0$. This completes the proof.

Theorem 2. If $p_{1}+q_{1}<0, p_{2}+q_{2}<0$, and $p_{2}^{2}-q_{2}^{2}<0$ hold, then (7) with $\tau=\tau_{n}^{+}$has a pair of pure imaginary roots $\pm i \omega_{+}$. If $p_{1}+q_{1}<0, p_{2}+q_{2}<0, q_{1}^{2}+2 p_{0} p_{2}-p_{1}^{2}>0, p_{2}^{2}-q_{2}^{2}>0$, and $\left(q_{1}^{2}+2 p_{0} p_{2}-p_{1}^{2}\right)^{2}>4 p_{0}^{2}\left(p_{2}^{2}-q_{2}^{2}\right)$ hold and $\tau=\tau_{n}^{+}\left(\tau=\tau_{n}^{-}\right.$, resp.), (7) has a pair of pure imaginary roots $\pm i \omega_{+}$( $\pm i \omega_{-}$, resp.).

Proof. Equation (14) shows that only one positive solution $\omega_{+}^{2}$ is possible if

$$
p_{2}^{2}-q_{2}^{2}<0
$$

then (7) with $\tau=\tau_{n}^{+}$has a pair of pure imaginary roots $\pm i \omega_{+}$.

Two positive solutions $\omega_{ \pm}^{2}$ are possible if

$$
\begin{gathered}
q_{1}^{2}+2 p_{0} p_{2}-p_{1}^{2}>0, \quad p_{2}^{2}-q_{2}^{2}>0 \\
\left(q_{1}^{2}+2 p_{0} p_{2}-p_{1}^{2}\right)^{2}>4 p_{0}^{2}\left(p_{2}^{2}-q_{2}^{2}\right) .
\end{gathered}
$$

Equation (12) shows that $\tau$, which corresponds to $\omega_{ \pm}^{2}$, is given by

$$
\begin{array}{r}
\tau_{n}^{ \pm}=\frac{1}{\omega_{ \pm}} \arctan \left(\frac{p_{0} q_{1} \omega_{ \pm}^{3}+\left(p_{1} q_{2}-p_{2} q_{1}\right) \omega_{ \pm}}{\left(p_{0} q_{2}-p_{1} q_{1}\right) \omega_{ \pm}^{2}-p_{2} q_{2}}\right)+\frac{2 n \pi}{\omega_{ \pm}} \\
n=0,1,2, \ldots ;
\end{array}
$$

then (7) has a pair of pure imaginary roots $\pm i \omega_{+}\left( \pm i \omega_{-}\right.$, resp.). This completes the proof.

Theorem 3. (i) If $p_{1}+q_{1}<0, p_{2}+q_{2}<0$, and $p_{2}^{2}-q_{2}^{2}<0$ hold, then the equilibrium $P_{1}^{*}$ of model system (4) is asymptotically stable for $\tau \in\left[0, \tau_{0}\right)$ and unstable for $\tau>\tau_{0}$; Hopf bifurcation occurs when $\tau=\tau_{0}$; that is, a family of periodic solution bifurcations from $P_{1}^{*}$ as $\tau$ passes through the critical value $\tau_{0}$.

(ii) If $p_{1}+q_{1}<0$ and $p_{2}+q_{2}<0$ hold and $\tau_{n}^{ \pm}$is defined by (18), then a positive integer $k$ exists such that $k$ switches from 
being stable to unstable and then to being stable again. In other words, when

$$
\tau \in\left[0, \tau_{0}^{+}\right),\left(\tau_{0}^{-}, \tau_{1}^{+}\right), \ldots,\left(\tau_{k-1}^{-}, \tau_{k}^{+}\right),
$$

the equilibrium $P_{1}^{*}$ of model system (4) is stable, whereas when

$$
\tau \in\left[\tau_{0}^{+}, \tau_{0}^{-}\right),\left(\tau_{1}^{+}, \tau_{1}^{-}\right), \ldots,\left(\tau_{k-1}^{+}, \tau_{k-1}^{-}\right),
$$

equilibrium $P_{1}^{*}$ is unstable. Therefore, bifurcations are present at $P_{1}^{*}$ when $\tau=\tau_{n}^{ \pm}, n=0,1,2, \ldots$.

Proof. To verify if bifurcations occur, the transversality conditions must be verified

$$
\frac{d}{d \tau} \operatorname{Re} \lambda\left(\tau_{n}^{+}\right)>0, \quad \frac{d}{d \tau} \operatorname{Re} \lambda\left(\tau_{n}^{-}\right)<0 .
$$

For this purpose, the following results are used [28]:

$$
\Theta=\operatorname{Sign}\left\{\frac{d}{d \tau} \operatorname{Re} \lambda\right\}_{\lambda=i \omega_{ \pm}}=\operatorname{Sign}\left\{\operatorname{Re}\left(\frac{d \lambda}{d \tau}\right)^{-1}\right\}_{\lambda=i \omega_{ \pm}} .
$$

Differentiating (7) with respect to " $\tau$," the following equation could be obtained:

$$
\left(\frac{d \lambda}{d \tau}\right)^{-1}=\frac{\left(2 p_{0} \lambda+p_{1}\right) e^{\lambda \tau}+q_{1}-\tau\left(q_{1} \lambda+q_{2}\right)}{q_{1} \lambda^{2}+q_{2} \lambda} .
$$

Thus,

$$
\begin{aligned}
\Theta & =\operatorname{Sign}\left\{\operatorname{Re}\left(\frac{d \lambda}{d \tau}\right)^{-1}\right\}_{\lambda=i \omega_{ \pm}} \\
& =\operatorname{Sign}\left\{p_{1}^{2}-q_{1}^{2}-2 p_{0} p_{2}+2 p_{0}^{2} \omega_{ \pm}^{2}\right\} \\
& =\operatorname{Sign}\left\{ \pm \sqrt{\left(q_{1}^{2}+2 p_{0} p_{2}-p_{1}^{2}\right)^{2}-4 p_{0}^{2}\left(p_{2}^{2}-q_{2}^{2}\right)}\right\} .
\end{aligned}
$$

The transversality conditions are satisfied. Therefore, $\tau_{n}^{ \pm}$are bifurcation values. This completes the proof.

\section{The Model with Time Delay within Fluctuating Environment}

Neglecting the second and higher powers of $x(t), y(t)$, and $E(t)$, the linearized system of model system (5) at the equilibrium $P_{1}^{*}$ could be obtained:

$$
\begin{gathered}
\dot{x}(t)=b_{11} x(t-\tau)+a_{12} y(t)+a_{13} E(t)+\xi_{1}(t), \\
\dot{y}(t)=a_{21} x(t)+a_{22} y(t)+\xi_{2}(t), \\
0=a_{31} x(t)+a_{33} E(t)+\xi_{3}(t),
\end{gathered}
$$

where $b_{11}=-\left(r_{1} / k\right) x^{*}, a_{12}=-\beta x^{*}, a_{13}=-x^{*}, a_{21}=$ $r_{2} y^{* 2} / \gamma x^{* 2}, a_{22}=-r_{2} y^{*} / \gamma x^{*}, a_{31}=p E^{*}$, and $a_{33}=p x^{*}-c$. Taking the Fourier transform of (25), the following system can be obtained:

$$
\widetilde{\xi}(s)=A(s) \widetilde{X}(s),
$$

where $\tilde{\xi}(s)=\left(\widetilde{\xi}_{1}(s), \widetilde{\xi}_{2}(s), \widetilde{\xi}_{3}(s)\right)^{T}, \widetilde{X}(s)=(\widetilde{x}(s), \widetilde{y}(s), \widetilde{E}(s))^{T}$, and

$$
\begin{aligned}
A(s) & =\left(\begin{array}{ccc}
i s-b_{11} e^{-i s \tau} & -a_{12} & -a_{13} \\
-a_{21} & i s-a_{22} & 0 \\
-a_{31} & 0 & -a_{33}
\end{array}\right) \\
& =\left(\begin{array}{lll}
A_{11} & A_{12} & A_{13} \\
A_{21} & A_{22} & A_{23} \\
A_{31} & A_{32} & A_{33}
\end{array}\right) .
\end{aligned}
$$

The following equation can be obtained:

$$
\widetilde{X}(s)=A^{-1}(s) \tilde{\xi}(s),
$$

where

$$
\begin{aligned}
A^{-1}(s) & =\left(\begin{array}{lll}
k_{11} & k_{12} & k_{13} \\
k_{21} & k_{22} & k_{23} \\
k_{31} & k_{32} & k_{33}
\end{array}\right) \\
& =\frac{1}{\operatorname{det}(A(s))}\left(\begin{array}{lll}
A_{11}^{*} & A_{21}^{*} & A_{31}^{*} \\
A_{12}^{*} & A_{22}^{*} & A_{32}^{*} \\
A_{13}^{*} & A_{23}^{*} & A_{33}^{*}
\end{array}\right),
\end{aligned}
$$

$A_{i j}^{*}$ are the algebraic cofactor of $A_{i j}$ and $i, j=1,2,3$.

If the function $Z(t)$ has zero mean value, the fluctuation intensity of the components in the frequency bands $s$ and $s+$ $d s$ is $S_{Z}(s) d s$, where spectral density $S_{Z}(s)$ is formally defined [29]. Consider

$$
S_{Z}(s) d s=\lim _{T \rightarrow \infty} \frac{\left\langle|\widetilde{Z}(s)|^{2}\right\rangle}{T} .
$$

Thus,

$$
\begin{aligned}
S_{\xi}(s) d s & =\lim _{T \rightarrow \infty} \frac{\left\langle|\tilde{\xi}(s)|^{2}\right\rangle}{T} \\
& =\lim _{T \rightarrow \infty} \frac{1}{T} \int_{-T / 2}^{T / 2} \int_{-T / 2}^{T / 2} \overline{\xi(t) \xi\left(t^{\prime}\right) e^{i s\left(t-t^{\prime}\right)} d t d t^{\prime} .}
\end{aligned}
$$

Therefore,

$$
\begin{aligned}
& S_{x}(s)=\sum_{j=1}^{3}\left|k_{1 j}\right|^{2} S_{\xi_{j}}(s), \\
& S_{y}(s)=\sum_{j=1}^{3}\left|k_{2 j}\right|^{2} S_{\xi_{j}}(s), \\
& S_{E}(s)=\sum_{j=1}^{3}\left|k_{3 j}\right|^{2} S_{\xi_{j}}(s)
\end{aligned}
$$


because $\left\langle\xi_{i}\right\rangle=0$ and $\left\langle\xi_{i}(t) \xi_{j}\left(t_{1}\right)\right\rangle=\delta_{i j} \delta\left(t-t_{1}\right), i, j=1,2,3$. Furthermore, $S_{\xi_{i}}(s)=1, i=1,2,3$. Thus, the fluctuation intensity for $x(t), y(t)$, and $E(t)$ is given by [29]

$$
\begin{aligned}
\sigma_{x}^{2} & =\frac{1}{2 \pi} \int_{-\infty}^{+\infty} S_{x}(s) d s \\
& =\frac{1}{2 \pi} \sum_{j=1}^{3} \int_{-\infty}^{+\infty}\left|k_{1 j}\right|^{3} S_{\xi_{j}}(s) d s \\
& =\frac{1}{2 \pi} \sum_{j=1}^{3} \int_{-\infty}^{+\infty}\left|k_{1 j}\right|^{3} d s, \\
\sigma_{y}^{2} & =\frac{1}{2 \pi} \int_{-\infty}^{+\infty} S_{y}(s) d s \\
& =\frac{1}{2 \pi} \sum_{j=1}^{3} \int_{-\infty}^{+\infty}\left|k_{2 j}\right|^{3} S_{\xi_{j}}(s) d s \\
& =\frac{1}{2 \pi} \sum_{j=1}^{3} \int_{-\infty}^{+\infty}\left|k_{2 j}\right|^{3} d s, \\
& =\frac{1}{2 \pi} \sum_{j=1}^{3} \int_{-\infty}^{+\infty}\left|k_{3 j}\right|^{3} d s . \\
\sigma_{E}^{2} & =\frac{1}{2 \pi} \int_{-\infty}^{+\infty} S_{E}(s) d s \\
& \frac{1}{2 \pi} \int_{j=1}^{+\infty}\left|k_{3 j}\right|^{3} S_{\xi_{j}}(s) d s
\end{aligned}
$$

After calculation, the fluctuation intensity for $x(t), y(t)$, and $E(t)$ is given by

$$
\begin{aligned}
& \sigma_{x}^{2}=\frac{1}{2 \pi} \sum_{j=1}^{3} \int_{-\infty}^{+\infty}\left|\frac{A_{j 1}^{*}}{\operatorname{det}(A(s))}\right|^{2} d s, \\
& \sigma_{y}^{2}=\frac{1}{2 \pi} \sum_{j=1}^{3} \int_{-\infty}^{+\infty}\left|\frac{A_{j 2}^{*}}{\operatorname{det}(A(s))}\right|^{2} d s, \\
& \sigma_{E}^{2}=\frac{1}{2 \pi} \sum_{j=1}^{3} \int_{-\infty}^{+\infty}\left|\frac{A_{j 3}^{*}}{\operatorname{det}(A(s))}\right|^{2} d s .
\end{aligned}
$$

Explicit values of the spectral densities of the populations and harvest effort when $\tau>0$ are difficult to obtain because evaluating the integrals is a difficult task. Numerical simulation shows that the intensity of fluctuation for the population and harvest effort from their steady state value increases gradually as the delayed parameter $\tau$ increases.

\section{Numerical Simulation}

This section presents the numerical simulation results of the delayed model system and stochastic delayed model system for different values of the delayed parameter $\tau$.
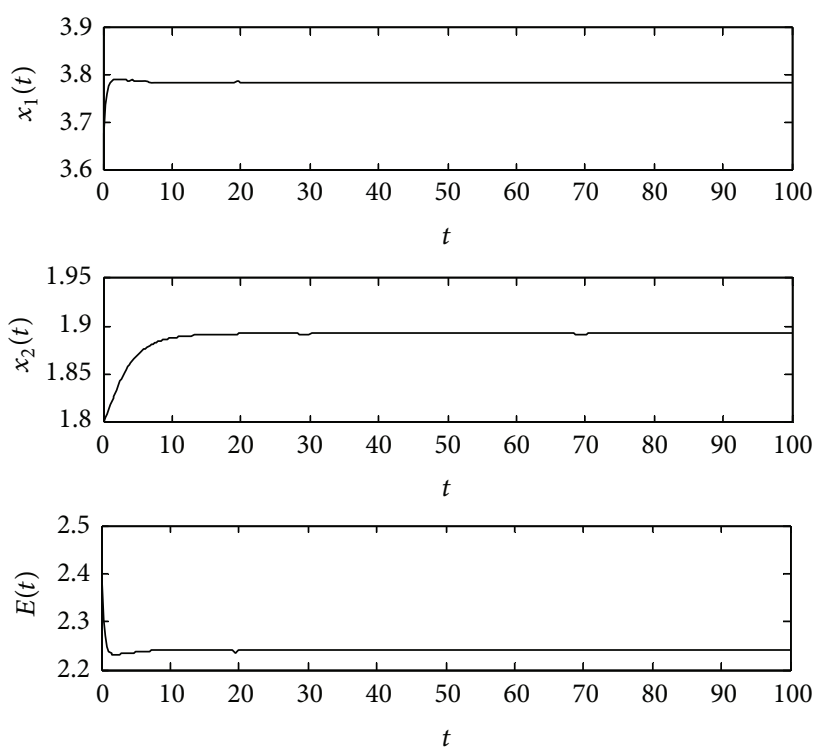

FIgURE 1: Stable dynamic response of system (4) at the interior equilibrium $P_{1}^{*}$ with $\tau=0.01$.

4.1. Numerical Simulation of the Delayed Model System. For model system (4), $r_{1}=10, r_{2}=0.3, k=5, \beta=0.1$, and $\gamma=0.5$. Furthermore, $p=0.5$ and $c=1$. Thus, $p r_{1}-c\left(r_{1} / k+\beta \gamma\right)>0$ is satisfied. Further calculations show that $m^{*} \approx 2.1226$. The analysis in Section 2 shows that two interior equilibria is possible when $0<m<m^{*}$; only one interior equilibrium is available when $m \approx m^{*}$; and an interior equilibrium is impossible when $m>m^{*}$. In the following part, we focus on the case where $0<m<m^{*}$ and the economic profit is set as $m=2$. Two interior equilibria can be obtained as follows: $P_{1}^{*}(3.7848,1.8924,2.2411)$ and $P_{2}^{*}(3.0932,1.5466,3.6589)$. Only the dynamical responses and corresponding phase portrait of model system (4) at $P_{1}^{*}$ are plotted; however, certain symmetric results about $P_{2}^{*}$ can be also obtained. The corresponding $\tau_{0} \approx 0.1512$ can be calculated by solving (18). Hence, the interior equilibrium remains stable for $\tau<\tau_{0}$, as shown in Figure 1. As $\tau$ increases, a periodic solution caused by Hopf bifurcation occurs, as shown in Figure 2. Figure 3 shows a limit cycle that corresponds to the periodic solution in Figure 2, which forms around the fixed point. Furthermore, Figure 4 shows that model system (4) remains unstable for sufficiently large $\tau$ but has complex structures with increasing oscillations.

4.2. Numerical Simulation of the Stochastic Delayed Model System. For the numerical simulation of the stochastic version corresponding to the delayed model system, the same set of parametric values was used. In the previous section, the analytical results for the population and harvest effort fluctuation around their steady states in the presence of discrete time delay and environmental forcing were obtained. However, the analysis cannot provide clear information about the stochastic stability behavior of the populations and the harvest effort. To answer these questions, the stochastic model system was simulated by increasing the magnitude of 

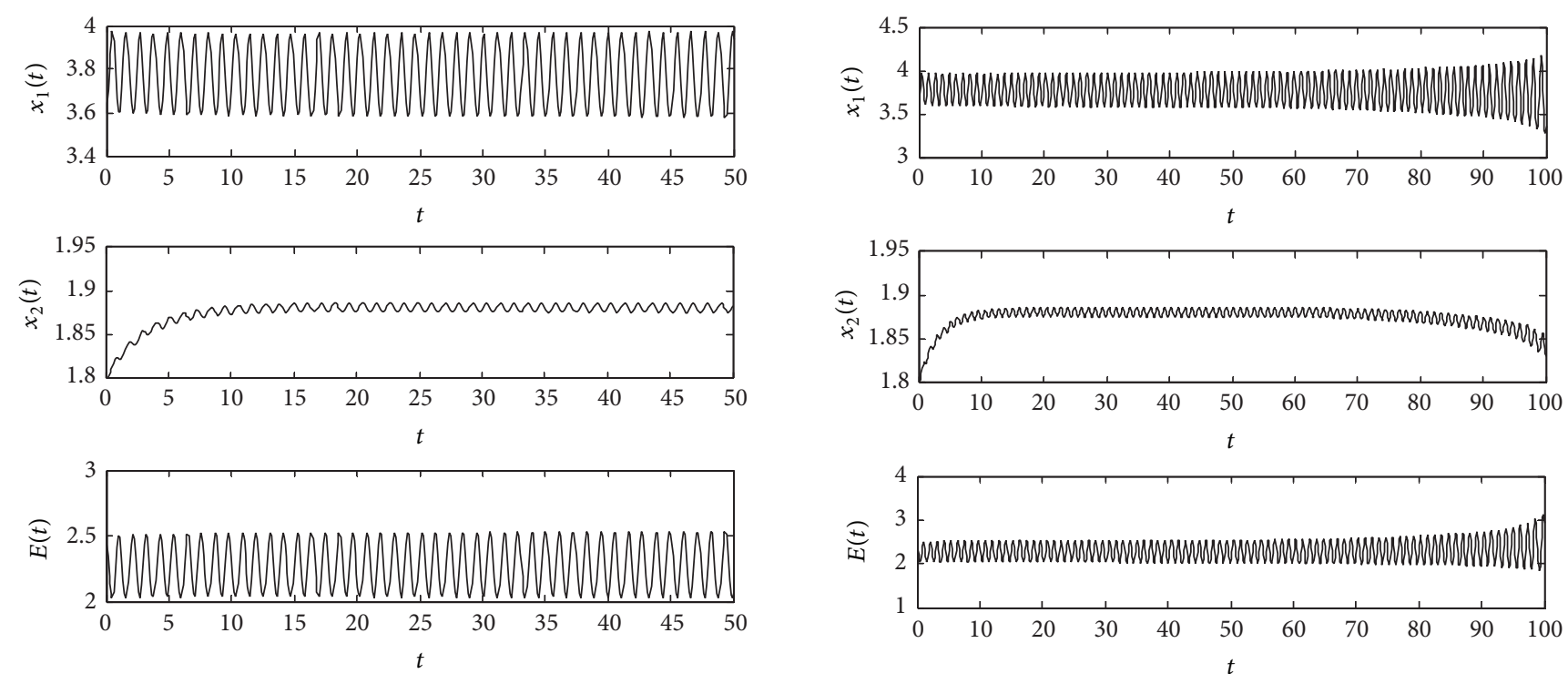

FIGURE 2: Periodic solution of system (4) at the interior equilibrium $P_{1}^{*}$ with $\tau_{0} \approx 0.1512$.

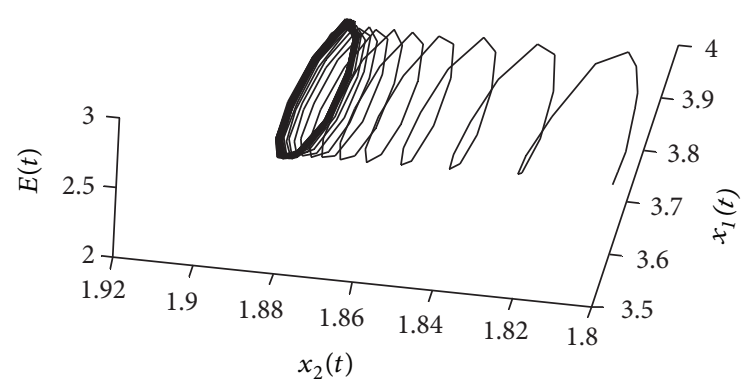

FIGURE 3: Limit cycle for system (4) corresponding to the periodic solution in Figure 2.

$\tau$ at each step. The amplitude of oscillation for $x(t), y(t)$, and $E(t)$ was enhanced compared with the oscillation observed in a deterministic environment. If the frequency of oscillation in $x(t), y(t)$, and $E(t)$ in Figures $1,2,3,5,6$, and 7 is compared, environmental fluctuation clearly plays a crucial role in determining the magnitude of oscillation (given that the magnitude of delay parameter is the same in both cases).

\section{Conclusion}

The effect of environmental fluctuations and time delay on the singular Leslie-Gower prey-predator bioeconomic model was investigated through theoretical analysis and numerical simulation. The local stability behavior around the interior equilibrium point of the delayed model system within deterministic environment is discussed, which extends the work done in [4]. The interior equilibrium switched from being stable to unstable and then back to being stable, as the time delay increases. The condition for Hopf bifurcation periodic solution was obtained by considering time delay as a bifurcation parameter. These analyses and numerical simulations show the sensitivity of the singular Leslie-Gower

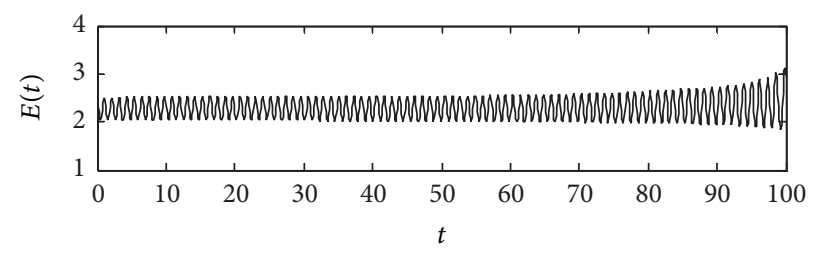

FIGURE 4: Unstable oscillation solution of system (4) at the interior equilibrium $P_{1}^{*}$ with $\tau=0.15122$.
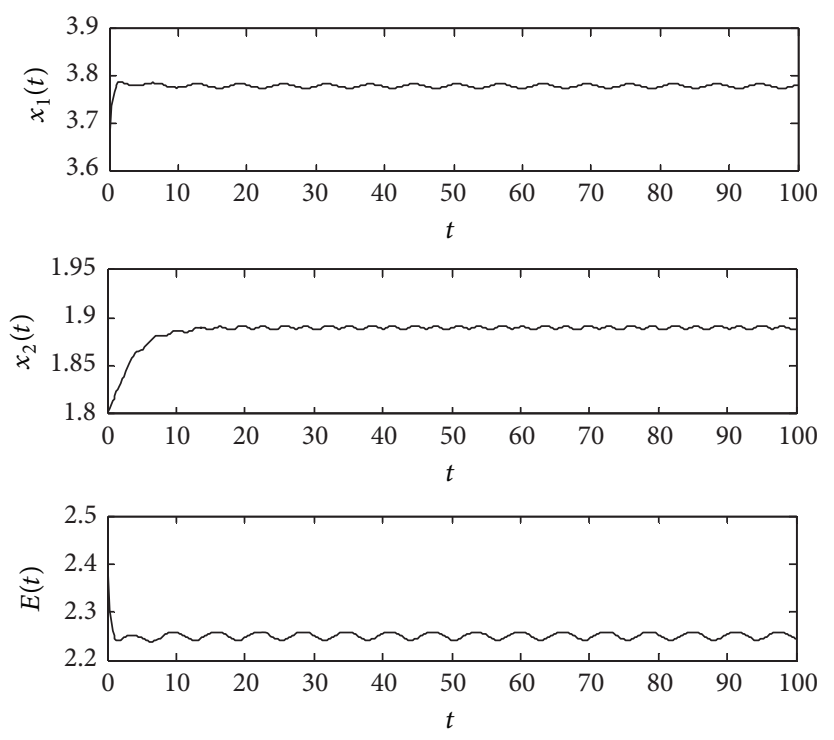

FIgURE 5: Periodic orbits of stochastic system (5) at interior equilibrium $P_{1}^{*}$ with $\tau=0.01$.

prey-predator bioeconomic model dynamics on time delay. Subsequently, the singular stochastic Leslie-Gower preypredator bioeconomic model with time delay was obtained by introducing Gaussian white noise terms to the deterministic model system mentioned above. The dynamic behavior of the stochastic model system around their steady state values produces drastic change with the presence of time delay and increasing magnitude. The frequency and amplitude of oscillation for the population density and harvest effort are enhanced relative to these values in the deterministic model system. These results indicate that the magnitude of time delay plays a crucial role in determining the stability 

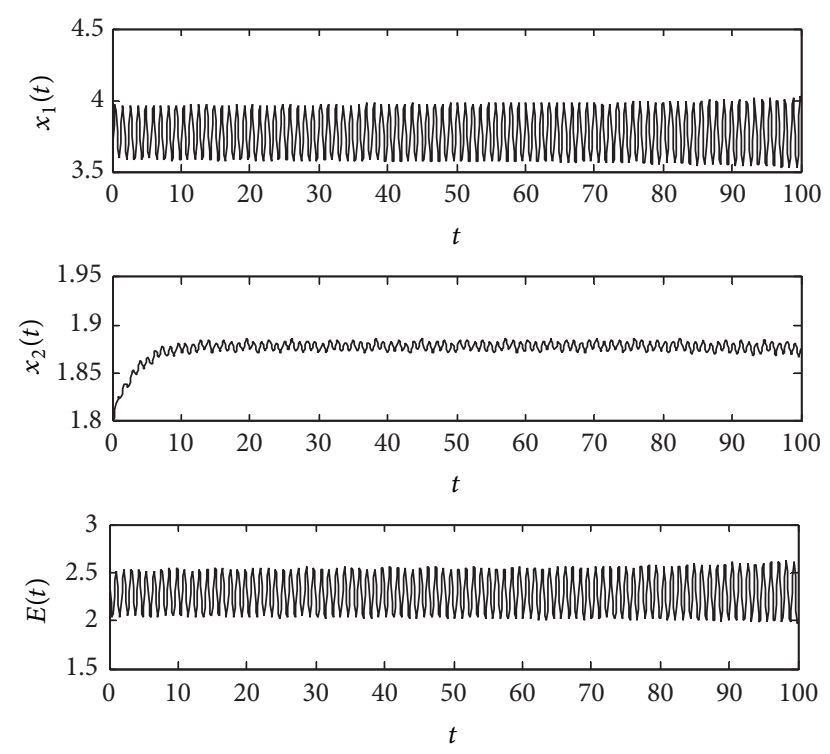

FIGURE 6: Oscillation orbits of stochastic system (5) at interior equilibrium $P_{1}^{*}$ with $\tau_{0} \approx 0.1512$.
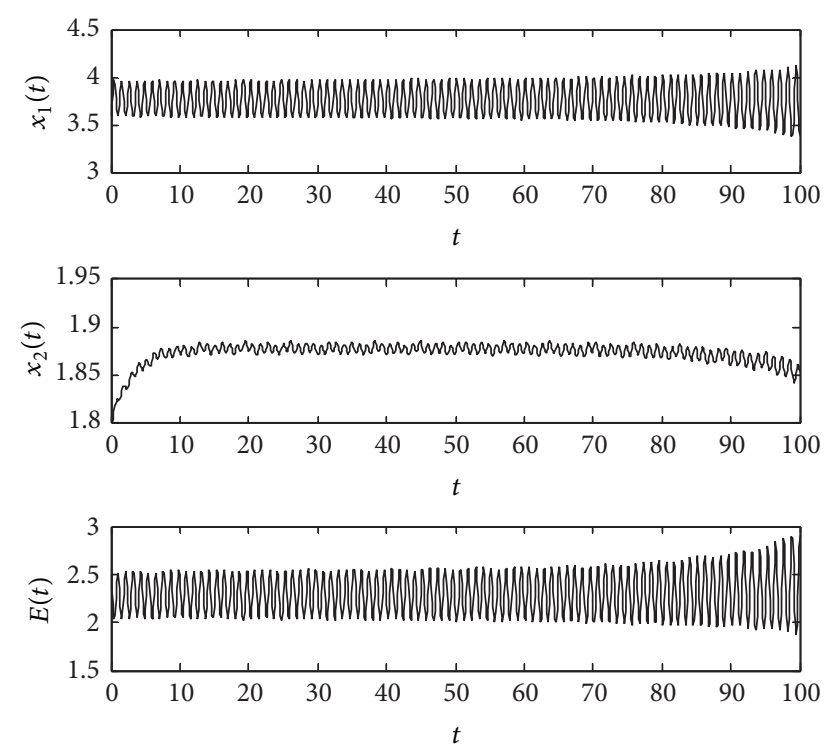

FIGURE 7: Oscillation orbits of stochastic system (5) at interior equilibrium $P_{1}^{*}$ with $\tau=0.15122$.

or instability. Environmental fluctuation intensity plays a crucial role in determining the magnitude of oscillation of the singular Leslie-Gower prey-predator bioeconomic model system within a fluctuating environment.

Notably, the differential-algebraic models presented in [13-17] investigated the problems of bifurcation and control on some bioeconomic systems with time delay. Compared with these works, the model proposed in this paper investigates the dynamical behavior of bioeconomic systems with stochastic fluctuations and time delay, which provides the work studied in this paper with new and positive features.

\section{Conflict of Interests}

The authors declare that there is no conflict of interests regarding the publication of this paper.

\section{Acknowledgments}

This work is supported by National Natural Science Foundation of China (61273008 and 61104003). The authors gratefully acknowledge the reviewers for their comments and suggestions that greatly improved the presentation of this work.

\section{References}

[1] M. Bandyopadhyay and S. Banerjee, "A stage-structured preypredator model with discrete time delay," Applied Mathematics and Computation, vol. 182, no. 2, pp. 1385-1398, 2006.

[2] Y. Qu and J. Wei, "Bifurcation analysis in a predator-prey system with stage-structure and harvesting," Journal of the Franklin Institute, vol. 347, no. 7, pp. 1097-1113, 2010.

[3] G. Liu and J. Yan, "Positive periodic solutions for neutral delay ratio-dependent predator-prey model with Holling type III functional response," Applied Mathematics and Computation, vol. 218, no. 8, pp. 4341-4348, 2011.

[4] S. Yuan and Y. Song, "Stability and Hopf bifurcations in a delayed Leslie-Gower predator-prey system," Journal of Mathematical Analysis and Applications, vol. 355, no. 1, pp. 82-100, 2009.

[5] G. Zhang, Y. Shen, and B. Chen, "Positive periodic solutions in a non-selective harvesting predator-prey model with multiple delays," Journal of Mathematical Analysis and Applications, vol. 395, no. 1, pp. 298-306, 2012.

[6] S. Devi, "Effects of prey refuge on a ratio-dependent predatorprey model with stage-structure of prey population," Applied Mathematical Modelling, vol. 37, no. 6, pp. 4337-4349, 2013.

[7] Y. Chen and F. Zhang, "Dynamics of a delayed predatorprey model with predator migration," Applied Mathematical Modelling, vol. 37, no. 3, pp. 1400-1412, 2013.

[8] A. Martin and S. Ruan, "Predator-prey models with delay and prey harvesting," Journal of Mathematical Biology, vol. 43, no. 3, pp. 247-267, 2001.

[9] H. Guo and L. Chen, "The effects of impulsive harvest on a predator-prey system with distributed time delay," Communications in Nonlinear Science and Numerical Simulation, vol. 14, no. 5, pp. 2301-2309, 2009.

[10] T. K. Kar and A. Ghorai, "Dynamic behaviour of a delayed predatorprey model with harvesting," Applied Mathematics and Computation, vol. 217, pp. 9085-9104, 2011.

[11] K. Chakraborty, S. Jana, and T. K. Kar, "Effort dynamics of a delay-induced prey-predator system with reserve," Nonlinear Dynamics, vol. 70, no. 3, pp. 1805-1829, 2012.

[12] C. Liu, Q. Zhang, J. Li, and W. Yue, "Stability analysis in a delayed prey-predator-resource model with harvest effort and stage structure," Applied Mathematics and Computation, vol. 238, pp. 177-192, 2014.

[13] X. Zhang and Q. 1. Zhang, "Bifurcation analysis and control of a class of hybrid biological economic models," Nonlinear Analysis. Hybrid Systems, vol. 3, no. 4, pp. 578-587, 2009.

[14] K. Chakraborty, M. Chakraborty, and T. K. Kar, "Bifurcation and control of a bioeconomic model of a prey-predator system 
with a time delay," Nonlinear Analysis: Hybrid Systems, vol. 5, no. 4, pp. 613-625, 2011.

[15] K. Chakraborty, M. Chakraborty, and T. K. Kar, "Optimal control of harvest and bifurcation of a prey-predator model with stage structure," Applied Mathematics and Computation, vol. 217, no. 21, pp. 8778-8792, 2011.

[16] G. Zhang, L. Zhu, and B. Chen, "Hopf bifurcation and stability for a differential-algebraic biological economic system," Applied Mathematics and Computation, vol. 217, no. 1, pp. 330-338, 2010.

[17] G. Zhang, B. Chen, L. Zhu, and Y. Shen, "Hopf bifurcation for a differential-algebraic biological economic system with time delay," Applied Mathematics and Computation, vol. 218, no. 15, pp. 7717-7726, 2012.

[18] R. M. Nisbet and W. S. C. Gurney, Modelling Fluctuating Populations, Wiley-Interscience, New York, NY, USA, 1982.

[19] T. Saha and M. Bandyopadhyay, "Dynamical analysis of a delayed ratio-dependent prey-predator model within fluctuating environment," Applied Mathematics and Computation, vol. 196, no. 1, pp. 458-478, 2008.

[20] M. Bandyopadhyay, T. Saha, and R. Pal, "Deterministic and stochastic analysis of a delayed allelopathic phytoplankton model within fluctuating environment," Nonlinear Analysis. Hybrid Systems, vol. 2, no. 3, pp. 958-970, 2008.

[21] T. K. Kar, "Influence of environmental noises on the Gompertz model of two species fishery," Ecological Modelling, vol. 173, no. 2-3, pp. 283-293, 2004.

[22] A. Elsonbaty, A. Elsaid, and H. M. Nour, "Effects of environmental fluctuation and time delay on ratio dependent hyperparasitism model," Communications in Nonlinear Science and Numerical Simulation, vol. 16, no. 6, pp. 2609-2619, 2011.

[23] B. Mukhopadhyay and R. Bhattacharyya, "Role of gestation delay in a plankton-fish model under stochastic fluctuations," Mathematical Biosciences, vol. 215, no. 1, pp. 26-34, 2008.

[24] H. Qiu, M. Liu, K. Wang, and Y. Wang, "Dynamics of a stochastic predator-prey system with Beddington-DeAngelis functional response," Applied Mathematics and Computation, vol. 219, no. 4, pp. 2303-2312, 2012.

[25] M. Liu, H. Qiu, and K. Wang, "A remark on a stochastic predator-prey system with time delays," Applied Mathematics Letters, vol. 26, no. 3, pp. 318-323, 2013.

[26] M. Liu and C. Bai, "Global asymptotic stability of a stochastic delayed predator-prey model with Beddington-DeAngelis functional response," Applied Mathematics and Computation, vol. 226, pp. 581-588, 2014.

[27] J. Dieudonné, Foundations of Modern Analysis, Academic Press, New York, NY, USA, 1960.

[28] K. L. Cooke and Z. Grossman, "Discrete delay, distributed delay and stability switches," Journal of Mathematical Analysis and Applications, vol. 86, no. 2, pp. 592-627, 1982.

[29] R. M. Nisbet and W. S. C. Gurney, Modelling Fluctuating Populations, Wiley Interscience, New York, NY, USA, 1982. 


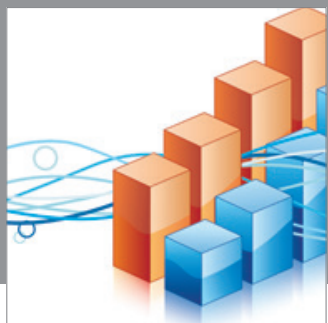

Advances in

Operations Research

mansans

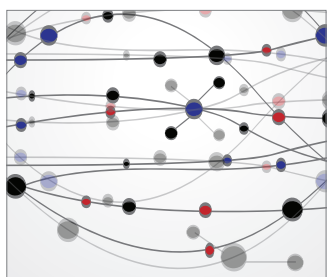

The Scientific World Journal
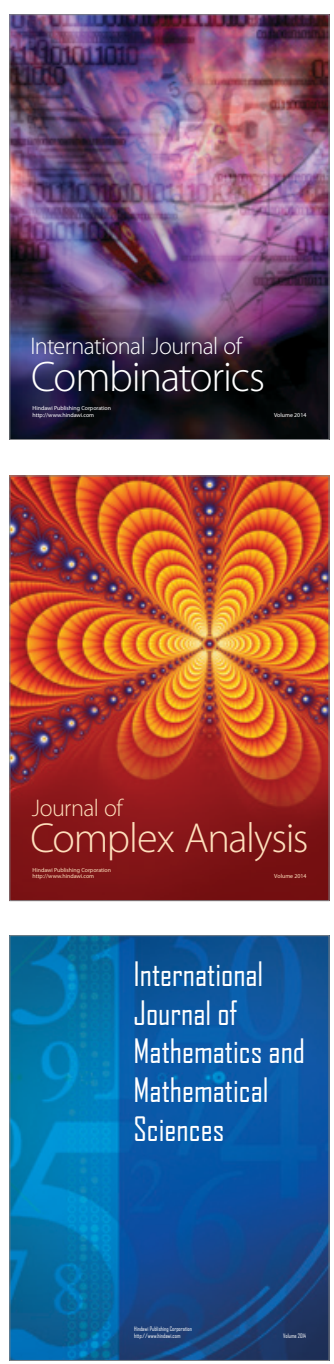
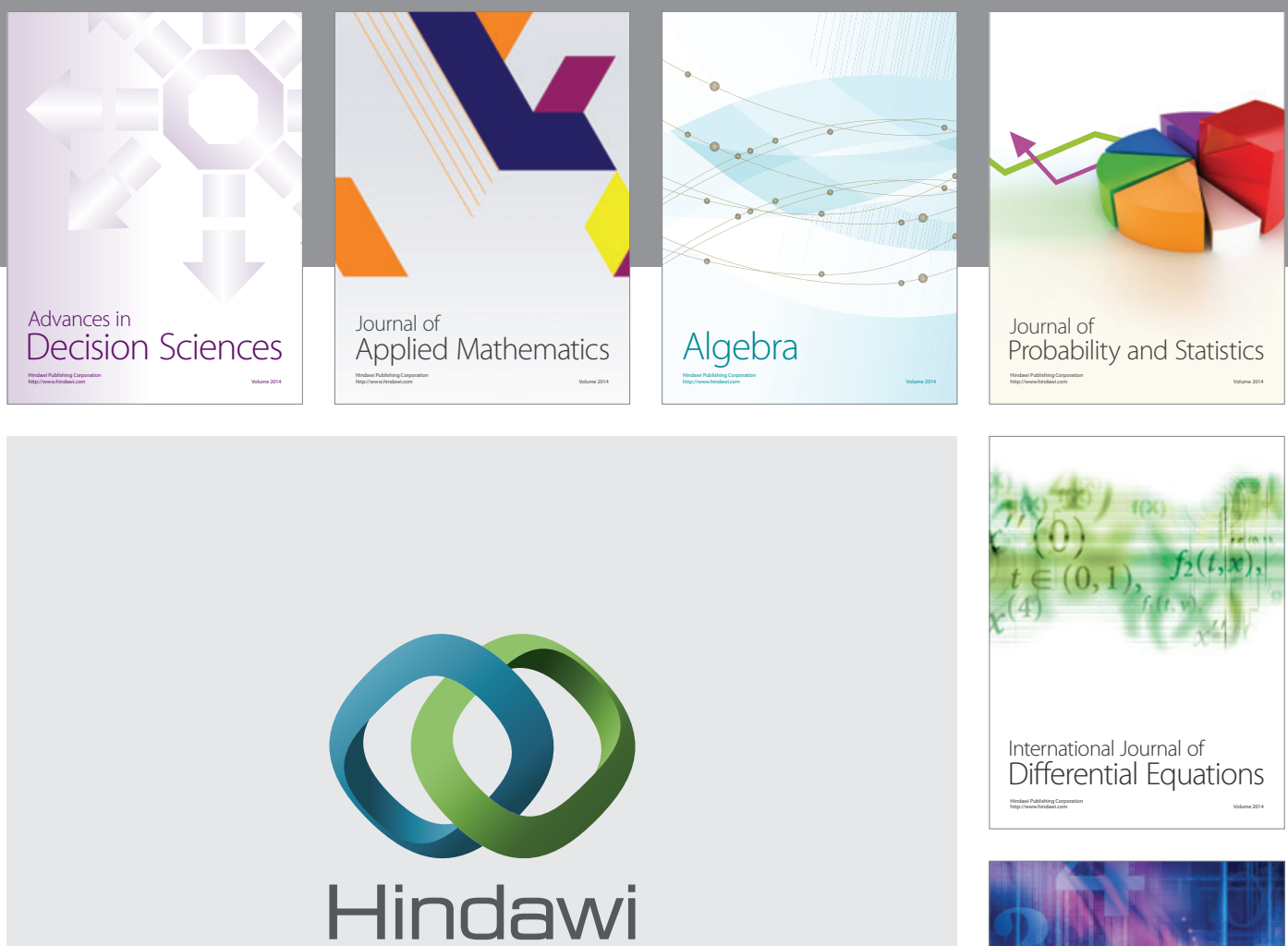

Submit your manuscripts at http://www.hindawi.com
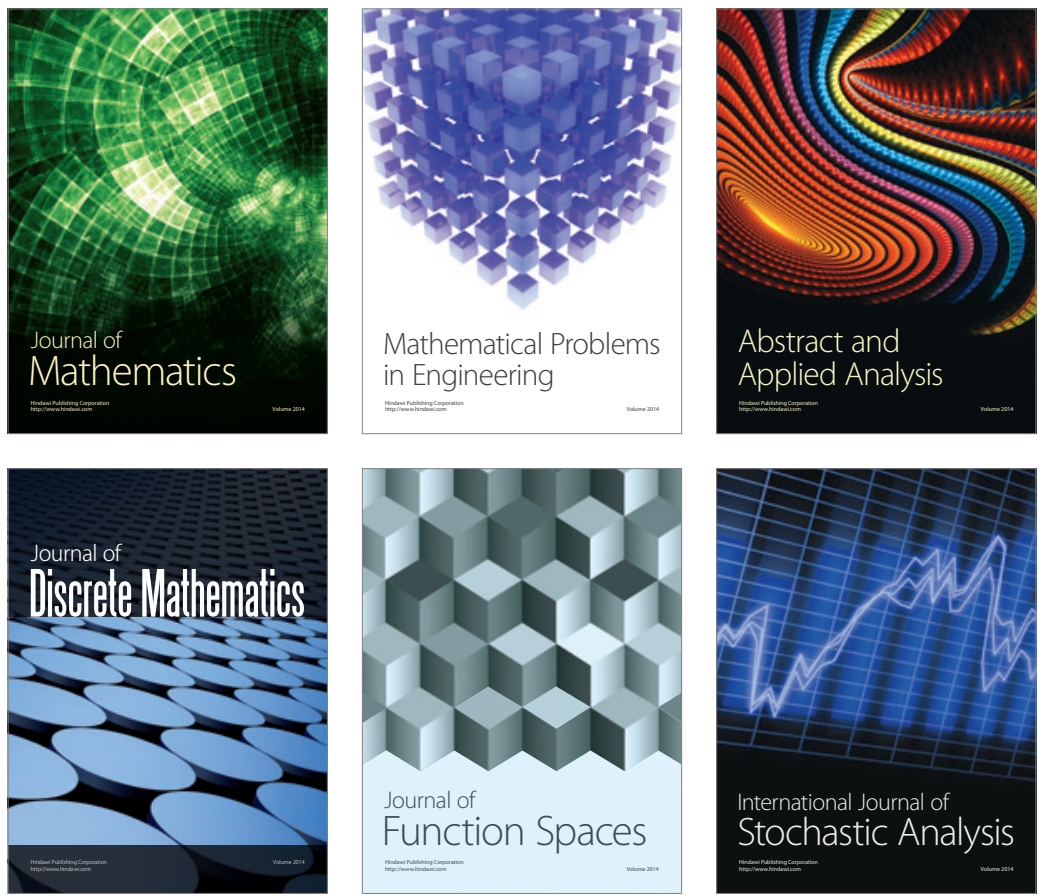

Journal of

Function Spaces

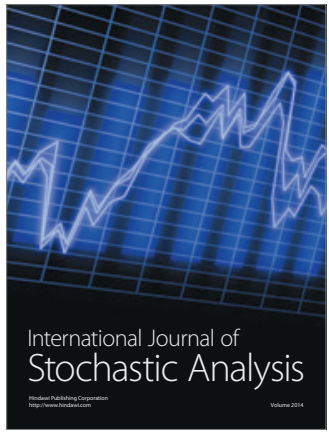

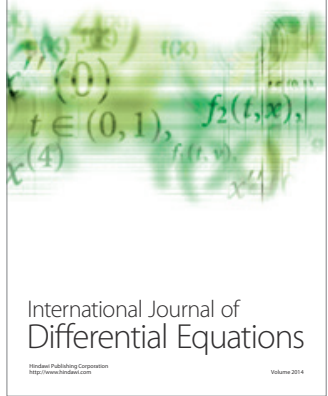
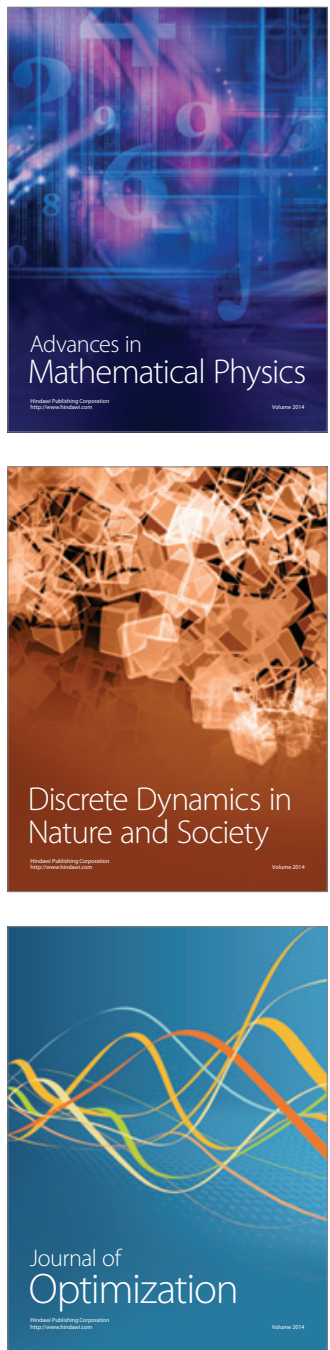